
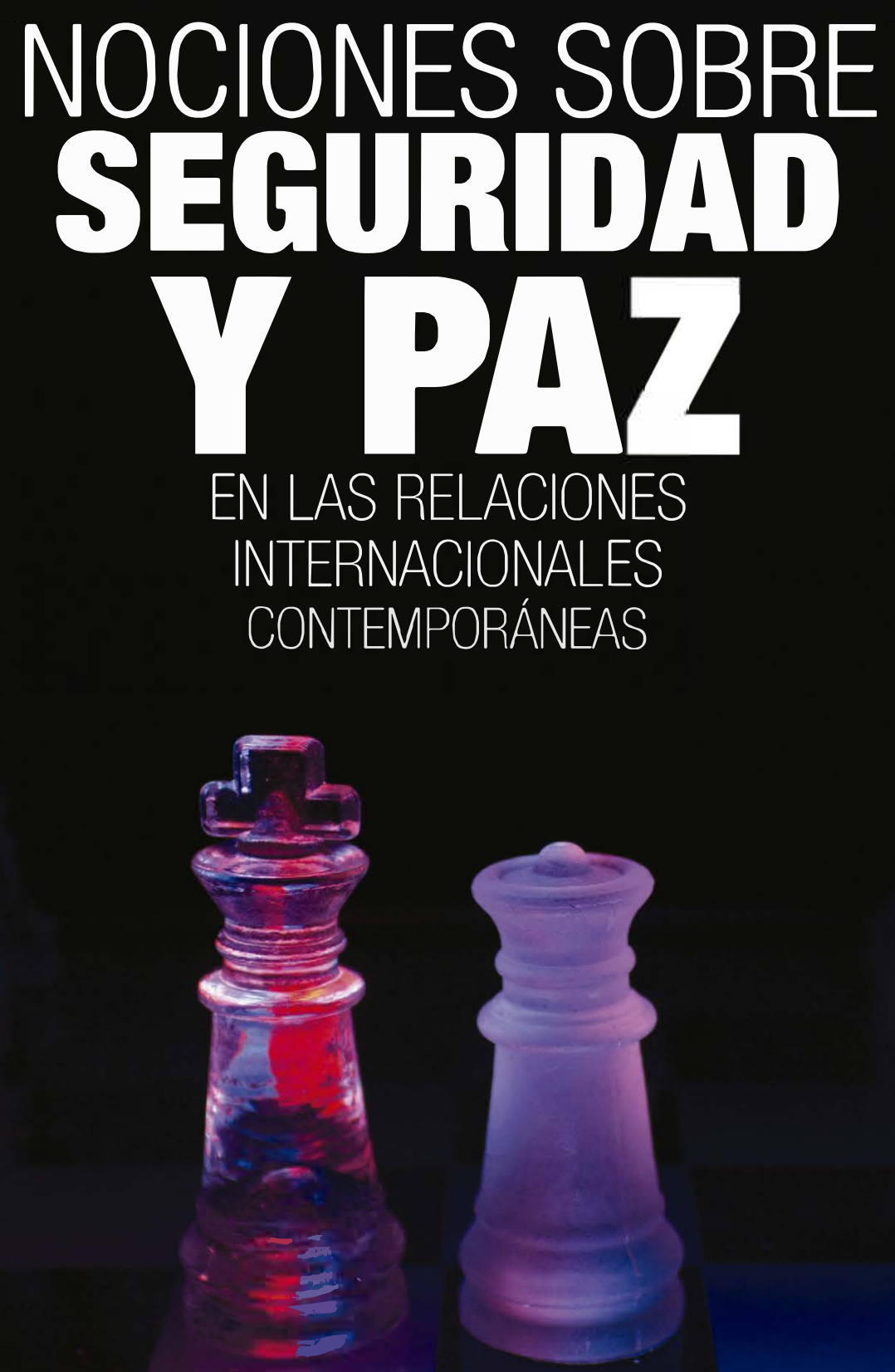

ALBERTO CASTILLO CASTAÑEDA CÉSAR AUGUSTO NIÑO GONZÁLEZ Editores

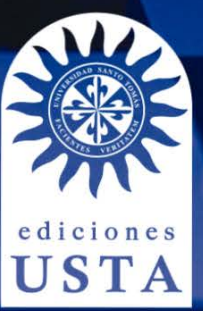




\title{
Nociones sobre seguridad y paz en las relaciones internacionales contemporáneas
}

\author{
Alberto Castillo Castañeda \\ César Augusto Niño González \\ EDITORES
}

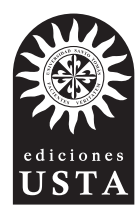


Nociones sobre seguridad y paz en las relaciones internacionales contemporáneas / Alberto Castillo Castañeda, César Augusto Niño González compiladores - editores, Bogotá: Universidad Santo Tomás, 2017.

549 páginas, ilustraciones, graficas

Incluye referencias bibliográficas

ISBN 978-958-782-021-8

1. Seguridad pública -- Colombia 2. Seguridad Ciudadana -- Colombia 3. Conflicto armado -- Colombia 4. Terrorismo I. Castillo Castañeda, Alberto II. Niño González, César Augusto III. Universidad Santo Tomás (Colombia).

CDD 303.609

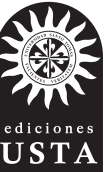

(C) Alberto Castillo Castañeda, Armando Borrero Mansilla, Andrés Gaitan, Catalina Monroy, César Augusto Niño González, Cristhian Sánchez, David González, Dulfary Calderón, Emilmar Rodríguez, Fabio Sánchez, Florent Frasson-Quenoz, Jerónimo Ríos, José Cepeda, Johanna Amaya, Juan Vallejo, Laura Ballén, Luis Aparicio, Mauricio Jaramillo, Mauricio Palma, Miguel Gomis-Balestreri, Miguel González, Nadia García, Néstor Maldonado, Néstor Rosanía, Roberto Brocate, Sara Quintero, Tania Gabriela Rodríguez Morales, Editores: Alberto Castillo Castañeda y César Augusto Niño

(C) Universidad Santo Tomás

Ediciones USTA

Carrera 9 n. ${ }^{\circ} 51-11$

Edificio Luis J. Torres, sótano 1

Bogotá, D. C., Colombia

Teléfonos: (+571) 5878797 ext. 2991

editorial@usantotomas.edu.co

http://ediciones.usta.edu.co

Directora editorial: Matilde Salazar Ospina

Coordinadora de libros: Karen Grisales Velosa

Coordinación editorial: María Carolina Suárez Sandoval

Asistente editorial: Andrés Felipe Andrade

Corrección de estilo: María Elvira Mejía Pardo

Diseño de carátula: Kilka Diseño Gráfico

Diagramación: CS2

Impreso en Colombia • Printed in Colombia

Impresión: Xpress Estudio Gráfico y Digital S.A.

ISBN 978-958-782-021-8

e-ISBN: 978-958-782-022-5

Primera edición, 2017

Hecho el depósito que establece la ley

Se prohíbe la reproducción total o parcial de esta obra, por cualquier medio, sin la autorización expresa del titular de los derechos. 


\section{Tabla de contenido}

Presentación XI

Castillo, Alberto y Niño, César

Prólogo XIII

FLORENT Frasson

REFLEXIONES Y APORTES CONCEPTUALES

La sexta generación de la guerra: entre degeneraciones y violencias en la seguridad internacional

NiÑo, CÉSAR

Las raíces del terrorismo moderno y su conexión con el mundo de las ideas. La relación entre nihilismo cultural y terrorismo político

ApARICIO, LUIS

Redefiniendo el paradigma de la seguridad y el terrorismo internacional: de la asimetría a la transnacionalidad

Ríos, Jerónimo y BRocAte, Roberto

La securitización del migrante como problema

García, NAdia

El derecho de intervención en el mundo de hoy

Borrero Mansilla, Armando 
Seguridad internacional mediante una aproximación

feminista: The WomanStats Project

Monroy, Catalina y Vallejo, Juan

El cambio medioambiental como un asunto de seguridad

y sus aportes a la consolidación de la paz territorial

BALlÉn, LAURA

Estados obstinados: ¿humanitarismo limitado?

La migración por asilo como parte de las agendas

de seguridad estatal y su choque con el régimen internacional de los derechos humanos

Palma, Mauricio

De la era de la disuasión a la era del control

GAITÁn, ANDRÉs

Objeción de conciencia en asuntos militares en el marco

de la seguridad y paz

Maldonado, Néstor

Los últimos de la fila: la reivindicación de la democracia en escenarios de posacuerdo

RosAnía, NÉstor

Seguridad y paZ en América Latina 307

La estrategia de seguridad en la Unasur 309

SÁNCHEZ, FABIo

Marco teórico para la inserción de Colombia en

América Latina y el Caribe en el escenario del posconflicto

GonzÁlez, DAvid y Rodríguez EMILMAR

Infancia excombatiente: lecciones de paz para

América Latina

Calderón, Dulfary

Política exterior y posconflicto: hacia una neutralidad activa en Colombia

Jaramillo, Mauricio 
Debilidad institucional y violencia en el Triángulo del Norte: hacia una gobernanza interna crítica con apoyo internacional

Amaya, Johanna; Cepeda, José y Gomis-Balestreri, Miguel

La evolución de la seguridad en el sistema internacional contemporáneo: un balance a partir de los casos de Estados Unidos y la Federación de Rusia

Quintero, Sara y González, Miguel

Política exterior rusa en un Nuevo Orden Mundial desde la mirada de Vladimir Putin

Rodríguez, TANiA

La extrema derecha: desafío para la integración y seguridad europea

SÁNCHEZ, CRISTHIAN

Epílogo

Castillo, Alberto

Autores 


\section{Presentación}

$\mathrm{Z}^{1}$ presente libro Nociones sobre seguridad y paz en las relaciones Cinternacionales contemporáneas es un esfuerzo colectivo por intentar repensar los conceptos clásicos de la seguridad y la paz en un mundo cambiante, dinámico y en constante transformación. En la Facultad de Gobierno y Relaciones Internacionales de la Universidad Santo Tomás, su Centro de Investigación, el Grupo de Estudios en Gobierno y Relaciones Internacionales, así como de la línea de Seguridad y Paz en Escenarios Transformados, nació la preocupación por reflexionar sobre los prismas y los lentes de análisis que inquietan a los académicos, los políticos y los tomadores de decisiones en materia de violencia, crimen, terrorismo, resolución de conflictos, construcción de paz, geopolítica, geoestratégia, estructuras armadas no convencionales, enfoques de género, medio ambiente y cambio climático, entre muchos más.

Entendimos que la seguridad y la paz son dos fenómenos que se encuentran circunscritos de manera transversal y constante en la historia de las naciones, de su desarrollo y evolución, o quizá "involución". Basados en esa misma lógica, vimos la necesidad de presentar un libro que demuestre dicha transversalidad intentando vislumbrar cuestiones críticas sobre los conceptos en mención.

De esa manera, este libro se convierte en una fuente de referencia para actuales y futuras investigaciones e invita a abordar los fenómenos 
desde nuevos enfoques, con reflexiones innovadoras desde el punto de vista de reconocidos académicos de Colombia y el extranjero. En este libro convergen áreas multifacéticas de estudio, corrientes teóricas, escuelas de pensamiento y lógicas científicas que le otorgan el valor agregado a esta obra.

Es una obra destinada al debate, la crítica y al rompimiento de viejos o tradicionales esquemas rígidos. Aquí parten nuevas dimensiones de análisis para los estudiantes y los investigadores, y promete ser el preludio de futuros trabajos de gran impacto académico. Estamos seguros de que las crisis son las oportunidades para repensar el mundo que habitamos.

\author{
Alberto Castillo Castañeda (Editor) \\ Decano Académico \\ Facultad de Gobierno y Relaciones Internacionales \\ Universidad Santo Tomás
}

César Augusto Niño González (Editor)

Director y profesor del Centro de Investigación Facultad de Gobierno y Relaciones Internacionales

Universidad Santo Tomás 